\title{
Circuit
}

Musiques contemporaines

\section{Différents sons de cloches}

\section{La parole aux artisans}

\section{Réjean Beaucage}

Volume 11, numéro 3, 2001

Perceptions

URI : https://id.erudit.org/iderudit/004650ar

DOI : https://doi.org/10.7202/004650ar

Aller au sommaire du numéro

Éditeur(s)

Les Presses de l'Université de Montréal

ISSN

1183-1693 (imprimé)

1488-9692 (numérique)

Découvrir la revue

\section{Citer cet article}

Beaucage, R. (2001). Différents sons de cloches : la parole aux artisans. Circuit, 11(3), 11-19. https://doi.org/10.7202/004650ar

\section{Résumé de l'article}

L'auteur s'est entretenu avec les 19 compositeurs de la Symphonie du millénaire, afin de recueillir leurs impressions sur le processus de création de l'oeuvre, sa création et les suites de cette aventure. Malgré les divergences d'opinions et les désaccords sur les façons de procéder, l'exercice aura permis à des compositeurs d'allégeances diverses de s'apprécier et d'unir leurs efforts dans l'écriture plurielle d'une oeuvre unique et singulière.
Ce document est protégé par la loi sur le droit d'auteur. L'utilisation des services d’Érudit (y compris la reproduction) est assujettie à sa politique d'utilisation que vous pouvez consulter en ligne.

https://apropos.erudit.org/fr/usagers/politique-dutilisation/ 


\section{Différents sons de cloches. La parole aux artisans}

Réjean Beaucage

[...] le simple fait que 19 compositeurs purent créer collectivement une ceuvre
d'une telle envergure sans se mutiler physiquement, ou aller se pendre après le
quatrième des très nombreux meetings, est digne de mention.

(Marie Pelletier)' $^{\prime}$

La Symphonie du millénaire, avant d'être, très probablement, le plus grand rassemblement populaire de l'histoire de la musique contemporaine, aura été l'occasion pour ses 19 artisans principaux de se livrer à un exténuant travail d'équipe, d'expérimenter les bons et les mauvais côtés de la création collective et, comme le faisaient les surréalistes par la pratique de jeux collectifs, «... [d'] ]aller au-delà de la subjectivité personnelle, [pour] découvrir où peut mener un discours dont les éléments proviennent de différents locuteurs. " (Durozoi, G. et Lecherbonnier, 1972, p 122.)

L'image romantique, fréquemment répandue, du compositeur se livrant en solitaire au labeur de la création en prend ici pour son rhume. Si plusieurs d'entre eux avaient déjà l'expérience du travail de groupe ${ }^{2}$, aucun des participants n'avait jamais pratiqué la création collective à une telle échelle. Avant même de songer à la mise en œuvre de l'opération concert, avec tout le travail de coordination de musiciens et de techniciens qu'un tel événement suppose, il fallait que 19 individualités puissent arriver à se fondre au sein d'une œuvre commune. Cela n'allait pas être le moindre des défis.

J'ai eu le plaisir, en ce début d'année 2001, de pouvoir correspondre ou discuter avec les 19 compositeurs de la Symphonie du millénaire afin de recueillir leurs souvenirs et impressions sur le processus de création, les résultats et l'éventuelle destinée de cette titanesque entreprise. Si pour certains d'entre eux l'événement peut sembler déjà bien lointain, d'autres pensent au contraire qu'il est encore trop frais pour que l'on puisse porter sur lui un jugement éclairé. Soit. Faisons tout de même un premier débroussaillage en espérant modestement qu'il
1. Marie Pelletier, compositrice participante, dans sa réponse au questionnaire de l'auteur. La plupart des citations des compositeurs et compositrices de la Symphonie ont ế recueillies par le biais d'un questionnaire écrit.

2. Dauphinais, Hamel et Lalonde forment le trio Espaces Sonores Illimités; Boudreau, Gonneville, Lalonde et Rea furent de laventure du Groupe des Sisses; Duchesne est I'un des fondateurs du collectif Ambiances Magnétiques; etc. 
pourra être utile, dans un avenir plus ou moins rapproché, à une analyse plus complète de l'œuvre.

\section{Conception}

On connaît assez maintenant l'anecdote de l'illumination qui frappa Walter Boudreau par un beau matin du début des années soixante et par laquelle il eut la vision quasi mystique d'une partition qui utiliserait tous les clochers et ensembles musicaux de Montréal. Le chemin allait toutefois se révéler long et sinueux entre le rêve et sa réalisation; ce n'est qu'au passage d'un millénaire à l'autre que seront réunies les conditions propices à l'élaboration d'un événement d'une telle envergure. Entre-temps, la vision purement esthétique de Boudreau se sera transformée en un projet comportant quatre objectifs précis :

1. Permettre à un nouveau public, beaucoup plus large que l'habituel public de la musique de création, de découvrir les compositeurs et leur travail par l'intermédiaire d'un projet populaire dans sa forme, mais au contenu hautement contemporain. L'alliage d'un lieu extramusical classique et d'une œuvre commune de compositeurs dits sérieux constitue une initiative novatrice;

2. Donner au monde de la création une visibilité exceptionnelle grâce au caractère de l'événement;

3. Créer un événement d'envergure dont les implications de coordination ef de production dépassent les intérêts individuels pour répondre à un besoin commun ;

4. Marquer l'an 2000 par un projet révélateur de l'esprit d'entreprise des organismes musicaux montréalais et de leur capacité à s'organiser pour réaliser un tel projet ${ }^{3}$

Je reviendrai sur les objectifs spécifiques de l'événement après avoir porté un regard sur ce qui en a tout de même constitué la matière première, soit l'œuvre présentée.

La première ébauche du projet, intitulée Esprits de clochers, faisait parader, à travers la ville, les ensembles musicaux (sur des chars allégoriques!) accompagnés en direct par les clochers croisés au fil de leur déplacement. L'électroacousticien Louis Dufort, recruté parmi les compositeurs, se montra assez sceptique sur les chances de réussite d'une telle aventure pour se mériter une place au comité technique :

Nous avons réalisé, assez rapidement, que cette idée était une utopie technique et qu'en fin de compte, la musique ne serait vraiment pas bien servie par cette

3. Tel que publié sur le site internet de la Symphonie du millénaire durant la période de préparation de l'événement. 
démarche. [...] nous avons finalement conclu qu'il nous fallait un site, un très grand site. La Providence nous a guidés vers l'Oratoire.

(Lovis Dufort)

Bien que les aspects techniques aient occupé une place de choix parmi les diverses préoccupations des organisateurs ${ }^{4}$, il va sans dire que la substantifique moelle de cet extraordinaire écheveau allait devoir être fournie par les compositeurs eux-mêmes. Là aussi, les idées de base allaient être appelées à évolver.

Au début il devait s'agir d'une œuvre collective beaucoup plus traditionnelle. Des œuvres de divers compositeurs qui s'enchaîneraient, [et] des transitions écrites collectivement. Puis, petit à petit, mouvement par mouvement, la Symphonie du millénaire s'est élaborée et est devenue ce projet à la démesure de la personnalité de ses concepteurs, à la surprise de bien des compositeurs du groupe. Quelques-uns d'entre nous se sont d'ailleurs sentis un peu piégés par l'aventure. Malgré tout, nous sommes tous restés à bord de ce vaisseau parfois houleux...

(Estelle Lemire)

Les directeurs artistiques du projet, Walter Boudreau et Denys Bouliane, se sont en effet rapidement retrouvés devant un véritable nœud gordien: l'édifice ne saurait être construit selon un plan dessiné par 19 personnes évoluant chacune à sa façon, comme dans une tentative de fédéralisme anarchique; d'un autre côté l'atteinte d'une parfaite harmonie à chacune des étapes du processus de création, dans la mesure même où l'on puisse l'imaginer, aurait requis un délai beaucoup plus considérable que ce qu'il était possible d'envisager. Peut-on vraiment décide a 19 d'un plan directeur qui saura satisfaire tout le monde? Les séances de travail n'étaient pas, dans ces circonstances, de tout repos, comme nous le rappelle Vincent Collard :

Le fait de travailler à 19 compositeurs n'allait pas de soi, c'est évident. 19 créateurs, 19 gros ego autour d'une même table - tirant la couverture, [...], s'affrontant sur des questions de principe, réclamant plus de démocratie, plus de cohésion, etc. rien n'était gagné d'avance. Plusieurs fois, j'ai cru que quelqu'un allait claquer la porte. De telles réunions, parfois fastidieuses, parfois excessivement houleuses, et ce toutes les deux ou trois semaines, c'était un peu épuisant psychologiquement.

Il fallait donc trancher. II fut alors décidé par l'ensemble du groupe de confier à Walter Boudreau et Denys Bouliane la tâche d'élaborer les principes structurels autour desquels allait s'articuler l'œuvre. "La planification générale élaborée par Denys Bouliane et Walter Boudreau était équilibrée et sécurisante. 》(Gilles Tremblay) Le plan préparé par les deux directeurs artistiques parut tout de même trop précis et contraignant au goût de certains des participants, donnant à quelques-uns l'impression de se transformer en coloristes, mais à la fin tous conclurent à sa nécessité. Ainsi, Yves Daoust a pu écrire
4. Notons au passage que le responsable de la sonorisation, Harvey Robitaille, recueille un concert d'éloges auprès des 19 compositeurs. 
Ce qui m'inquiétait surtout c'était la question de la méthode de travail : dans quelle mesure pourrions-nous vraiment arriver à une ceuvre collective, et non à un patchwork de blocs individuels. La solution est venue de l'imposition de structures d'interventions "coercitives", préparées par les directeurs artistiques. Cette approche, qui limitait le risque, a eu l'avantage de nous permettre d'être efficaces.

\section{Et Sean Ferguson}

Je crois que les compositeurs qui ont pu trouver le processus le plus satisfaisant son ceux qui ont pu laisser de côté leur désir de contrôler chacun des aspects de l'œuvre, ce qui est difficile à faire pour un compositeur. Chacun devait laisser son ego au vestiaire

La décision d'introduire le thème de l'hymne Veni Creator Spiritus comme cantus firmus allait évidemment se révéler déterminante en donnant à l'œuvre son épine dorsale. Selon Gilles Tremblay, "l'emploi de la mélodie grégorienne du Veni Creator a été un facteur de cohésion et un fil d'Ariane à travers toute l'œuvre, tant au plan microscopique que macroscopique ". Walter Boudreau confirme qu'il était important, étant donné le caractère populaire de l'événement, d'utiliser un thème relativement simple que les compositeurs auraient ensuite tout le loisir de triturer à leur manière et dans leur style propre. II s'agissait pour le public, aux yeux du codirecteur artistique, d'un exercice de psychologie cognitive appliquée! Denys Bouliane estime quant à lui que l'esprit de la création doit être un thème assez rassembleur pour des gens qui font de la création leur métier! Malgré tout, certains des participants auront trouvé le thème trop prépondérant, Luc Marce allant jusqu'à se demander, à la blague, s'il avait été embauché comme compositeur ou comme orchestrateur.

La thématique religieuse de l'œuvre, inspirée autant par le lieu de diffusion de l'événement que par l'histoire montréalaise, et l'étincelle initiale du projet de Boudreau (les clochers), allait servir de base à la construction de la Symphonie et à son découpage en sections nommées Appel, Enfer, Purgatoire, Contemplation/Aurores boréales, Paradis, Ascension, Apothéose et Épilogue, des termes qui ont une signification religieuse très forte mais qui portent en eux des sens différents, acquis au cours du millénaire. John Rea estime qu' «en dépit d'elle-même, l'œuvre dans sa forme et son contenu se révélait au public telle une action liturgique, à la fois sacrée et profane». Les compositeurs n'ont, par ailleurs, guère exprimé d'opinions sur cet aspect particulier de l'œuvre dans leurs réponses à mes questions. Seul Jean Lesage a fait connaître un point de vue dissident sur la chose: "La symbolique catholique romaine m'a toujours semblé inutile, dès le début du projet. Je n'ai pas changé d'idée à ce sujet. »

En agissant comme architectes de cette gargantuesque entreprise, les directeurs artistiques se sont-ils trop approprié l'œuvre? Peut-on prétendre qu'elle soit moins collective du fait que les directeurs artistiques aient accepté le mandat de la doter d'un squelette? Si cette question a pu être soulevée par certains parti-
5. «I think the composers that found the process the most satisfying were those who were able to give up their need to control for compect of the piece, which is difficult or composers. One ego at the door"." 
cipants, ce n'est certes pas le point de vue de la majorité. Sans doute, le sentiment de ne pas être maître du choix de la contrainte a pu, par moment, propager une certaine insécurité parmi les compositeurs. Le créateur, quelle que soit sa discipline, travaille toujours en fonction d'une contrainte, grande ou petite, qu'il s'impose à lui-même. L'imposition d'une contrainte par quelqu'un d'autre tend à établir un ordre hiérarchique implicite qui peut certes provoquer un stress. Comme l'explique Marie Pelletier :

C'était un climat très aléatoire. On ne savait jamais où l'on allait réellement, malgré la grande forme préexistante 6 . J'avais le sentiment que, dans le profil narratif total de l'œuvre, je n'avais pas réellement de vue d'ensemble , ce qui n'est pas le cas dans mon propre travail en général. Cette absence de contrôle fut déstabilisante, mais très stimulante, car cela me conduisit à un abandon, une certaine lascivité, presque contemplative, sur le plan de mon propre langage.

Tout le monde ne ressent pas forcément le même plaisir devant une contrainte extérieure; cela semble être le cas d'André Hamel, qui reconnaît tout de même la pertinence de l'exercice :

Walter et Denys avaient un rôle prédominant au niveau de la forme et de l'articulation des interventions de chacun. Les choses étaient, de prime abord, discutées de façon collective ${ }^{8}$ et, par la suite, Walter et Denys préparaient ce qu'il était convenu d'appeler une partition des interventions et ce, pour chacune des sections de l'œuvre. Ces partitions, de très libres, pour Le Purgatoire 13 minutes avec très peu de contraintes, sauf pour ce qui est du choix des autres groupes [instrumentaux] auxquels nous pouvions faire appell, sont devenues, à mesure que le travail progressait, de plus en plus contraignantes. Tant et si bien que pour ce qui est des dernières sections à avoir été écrites, notamment la Contemplation, L'Ascension et l'Apothéose/Épilogue, le plaisir s'est, disons, quelque peu dissipé. Peutêtre Walter et Denys ont-ils privilégié ce genre d'approche parce qu'ils ont jugé qu'il fallait qu'il y ait des passages avec plus d'unité. À cet égard, le résultat musical semble leur donner raison.

Il apparaît évident, à l'écoute, qu'un tel travail ne saurait être le fruit d'un seul créateur (ou même d'un duo). La richesse des arrangements et le foisonnement des styles appellent l'image du travail de groupe. L'extrême vitalité de l'ensemble est aussi, certainement, la conséquence directe d'une écriture plurielle. Ainsi, comme le fait remarquer Sean Ferguson:

Je crois que si je devais critiquer un aspect du résultat final, c'est qu'il y a très peu de moments de repos, de relâchement de la tension. Je crois que c'est dû au fait que les compositeurs étaient trop conscients du regard que leurs collègues pouvaient jeter par-dessus leur épaule?.

Nombreux sont les commentaires qui appuient cet aspect pluriel de l'œuvre, tel celui-ci

Avant tout, c'est une ceuvre unique en ce sens qu'il est absolument impossible qu'une telle pièce ait pu être écrite par une seule personne. Mais la diversité des styles et
6. II faut comprendre que, malgré la strucure établie et bien que le squelette ait pu procurer une forme assez claire à l'œuvre en devenir, chacun des compositeurs avait à travailler à un niveau microscopique et que son "obligation " d'interagir avec ses colègues, aussi stimulante puissetello être, ne égues, aussi stimulante puisse-telle être, ne vi laissait guère le loisir de planifier ses pro-

7. À rapprocher du sentiment éprouvé par Alain Dauphinais : "À un certain moment, i'ai accepté que je devais fabriquer des petits morceaux de sons, dont l'emplacement dans le grand tout avait été préalablement déterminé, et j'étais comme un artisan qui devait concevoir des petites pierres qu allaient éventuellement prendre place dans une mosaïque dont la globalité m'échappait encore.

8. Sur la qualité strictement "collective» de l'œuvre, Gilles Tremblay a une opinion aussi rranchée... qu'ouverte : «La Symphonie du millénaire a été un travail collectif et même une oeuvre collective let i'y inclus non seulement les 19 compositeurs, mais tous les artsans qui y ont participé). Par contre, dans toutes les étapes de son élaboration et a tous les niveaux, s'agitil vraiment d'une création collective? Une création collective este-lle possible? Ma réponse est un point d'interrogation. ”

9. «I think that if there is something I would criticize about the final result, it is that there are very few moments of repose or relaxare very few moments of repose or relaxarion of tension. I hink inis is maybe the result of composers being overly conscious of their colleagues looking over their shoul-
ders” 
des approches compositionnelles ne fait pas pour autant de la Symphonie du millénaire une ceuvre de collage. Cela est sûrement dû en partie à l'utilisation d'un thème commun Neni Creator), mais il y a autre chose qui donne une cohérence à l'œuvre. Cela reste indéfinissable. Peut-être est-ce l'air du temps? Peut-être est-ce notre "québécitude» commune?

(André Hamel)

Je ne peux m'empêcher de rapprocher cette citation d'André Hamel de cette autre, de Vincent Collard

La "promiscuité des styles 10 ", pour reprendre votre expression, a sans doute crée une certaine appréhension chez chacun d'entre nous, y compris Denys et Walter probablement. Mais le plan de l'œuvre était conçu de façon à les fondre harmonieusement ou, selon le cas, à les faire judicieusement contraster. De ce point de vue, je crois que le résultat est très réussi : la Symphonie a mis en lumière l'extraordi naire diversité stylistique de la musique québécoise, mais aussi, selon moi, une certaine Quebec touch qui cimentait le tout.

Serge Arcuri estime que la "promiscuité des styles de chacun était une donnée inhérente au projet et représentait le principal défi. Toutefois, le métissage étant une grande tendance dans l'écriture de cette fin de siècle, cela a permis la concrétisation d'un projet qui n'aurait sûrement pas pu se réaliser il y a vingt-cing ou trente ans». Hic et nunc!

Il faudrait sans doute davantage d'aventures collectives de cette dimension pour mesurer la faisabilité d'autres méthodes de travail et arriver enfin à définir ce qui caractérise la musique contemporaine d'ici. Ce ne sont certes pas les mélomanes qui pourraient s'en plaindre!
10. J'utilisais cette expression dans le questionnaire soumis à lensemble des compositeurs et compositrices. Gilles Tremblay préfère quant à lui une autre image : «La juxtaposition des styles n'en faisait pas une promiscuité, mais un voisinage poétique très riche, où la distance stylistique correspondait à l'ampleur de l'espace physique."

\section{Aboutissement}

On pourrait croire, d'après les propos que j'ai pu recueillir, que le plus durable souvenir que conserveront les compositeurs de la Symphonie du millénaire es celui d'une enfilade de réunions! Comme le souligne Jean Lesage

Je ne connais pas le jugement de l'histoire, et il est probablement trop tôt pou estimer si l'événement aura un impact durable. Cependant, ce qui m'a le plus marqué dans ce projet, ce n'est pas, curieusement, son aspect artistique comme son aspect humain. Le travail, les échanges, le partage avec mes collègues compositeurs m’ont marqué à jamais. Je me sens privilégié d'appartenir à leur communauté.

André Duchesne a, quant à lui, été vivement impressionné par l'événement et la très grande qualité de la réalisation technique, mais lors de notre discussion ses 
premiers souvenirs vont au "sentiment de respect que chacun portait au travail des autres. Les réunions étaient longues et nombreuses, mais c'était sympathique d'apprendre à se connaître». E† Marie Pelletier de renchérir :

La Symphonie du millénaire m'a permis d'entrer en relation avec mes collègues, à un niveau profond et intime, mais muet, par le biais de l'analyse de leurs écritures. Une analyse dynamique, qui sollicitait une réponse immédiate, à travers ma propre musique.

On comprend aisément à lire ces quelques lignes que l'idée d'intituler l'œuvre Esprits de clochers ait pu être abandonnée!

Cette collégialité a-t-elle permis aux participants d'obtenir une image claire de ce qu'allait être la Symphonie durant la préparation de ce grand "jam de 19 compositeurs vivants dont les règles ponctuelles ont d'ailleurs muté plusieurs fois »? (Anthony Rozankovic)

Quiconque prétendrait avoir eu une idée juste de ce que donnerait le processus avant le début des travaux manquerait sans doute de franchise. Même Walter et Denys ne pouvaient pas vraiment prévoir le résultat final avant d'y assister. [...] Pou être honnête, nous n'avions aucune idée de ce que serait le résultat musical final avant le soir du concert. C'était à la fois stimulant et terrifiant ${ }^{11}$.

(Sean Ferguson)

On comprend bien sûr que la lourdeur de l'infrastructure nécessaire ne permettait guère de tester les idées sur le terrain! La seule véritable répétition générale, tenue la veille de l'événement, n'aura même pas permis d'entendre l'œuvre dans son ensemble... Dans ces conditions, évidemment, l'imagination des compositeurs, placés devant une situation parfaitement inédite, devait être sérieusement mise à contribution. Mais comme le note l'électroacousticien Louis Dufort :

Normalement, je suis en contact direct avec la matière sonore alors que cette fois-ci i'allais entendre ce que ie composais seulement à la représentation de l'œeuvre. Certes, i'avais une petite idée, mais il est difficile d'imaginer un cluster de 200 instruments éparpillés sur un site d'un kilomètre carré. C'était en ce sens très excitant d'entendre ce que j'avais écrit pour les différents ensembles. J'ai eu de très belles surprises.

l'événement-concert du 3 juin 2000 constitue une production distincte de l'œuvre musicale qui pourrait lui survivre. II comportait des objectifs particuliers, que i'ai décliné au début de cet article. Si Gilles Tremblay parle d'un "événement musical majeur [...], et majeur au plan international», on doit tout de même constater que, à ce stade-ci du moins, ce sont souvent le gigantisme et le caractère testif de cette soirée du 3 juin 2000 qui semble avoir marqué tant le public que le groupe des 19, davantage que l'aspect purement musical de l'aventure. Ainsi, Yves Daoust peut-il lancer : "Pour moi ce n'est pas une œuvre, mais un événement. À mon avis très réussi ». À propos de la démesure de l'événement, Luc Marcel avouera même avoir cru s'être trompé de site tant il ne s'attendait pas à y
11. "Anyone who suggests that they had any real idea of what the process would be like before it began is perhaps being disingenuous. Even Walter and Denys couldn't have really predicted the final result before it was done. [...] To be honest, we really did $n^{\prime}$ t have any idea of what the final musical result would be until the actual performance. This was both exhilarating and terrifying.». 
trouver une telle assistance! II insiste également, comme la plupart des membres du groupe, sur l'aspect historique de ce concert. On peut sans doute penser qu'une analyse musicale objective de l'œuvre ne pourrait être réalisée que par une personne qui n'aurait pas assisté à l'événement, tant l'impact de son dévoilement en direct fut impressionnant.

II est indubitable que les quatre objectifs fixés par les promoteurs de l'événement ont été atteints, sinon dépassés, et ce au-delà de toute attente. Comme le souligne Estelle Lemire :

Ce projet a été deux choses à mon avis. D'abord une grande fête de la musique, une fête des compositeurs vivants, des compositeurs d'ici. Pour la plupart des gens, un compositeur de tradition classique c'est un nom étranger une personne d'un autre siècle. C'est Bach, Mozart, Beethoven ou Vivaldi. [...] Cet événement a changé un peu cette perception mythique de ce qu'est un compositeur et de ce que sont les compositeurs d'ici, en les représentant par un certain nombre d'entre eux, en montrant la diversité des styles et l'indépendance artistique des compositeurs montréalais. De plus, c'était un défi logistique et technique monstrueux, relevé haut la main et, je dois le dire, à la grande surprise de plusieurs d'entre nous.

Ce à quoi Vincent Collard ajoute :

[L'événement] a donné à 19 compositeurs de musique contemporaine la chance d'être entendus et ovationnés par des dizaines de milliers de personnes. Quand on pense qu'il est en général très difficile d'en déplacer 500, ce n'est pas si mal! [...] Et puis, depuis juin dernier, quand i'essaie d'expliquer ce qu'est la musique contemporaine à quelqu'un qui n'en sait rien, je dispose enfin d'une référence qu'il a une chance de connaître, ne serait-ce que par ouï-dire. C'est déjà un pas de géant!

Et les suites? Il y a eu les deux soirées au Planétarium de Montréal où l'on a pu entendre l'œuvre dépouillée de son caractère événementiel. Une tout autre approche du phénomène musical, que l'on voudrait certes pouvoir répéter à la maison tant l'œuvre est dense et exige une attention soutenue. Mais encore? Denys Bouliane et Walter Boudreau, qui louent l'investissement majeur consenti par les compositeurs et se félicitent à bon droit de la tâche accomplie, se montrent assez amers quant à la qualité de la couverture médiatique qui a été faite de l'événement. Presque rien au Canada anglais et finalement assez peu de choses dans les médias d'ici. Outre les articles des journalistes spécialisés des grands quotidiens, la majorité des médias ont couvert l'événement comme s'il s'agissait d'un spectaculaire accident ou d'un gros incendie (la Symphonie a fait la une des quatre grands quotidiens montréalais, mais bien peu d'articles de fond). L'ampleur et le nombre de «victimes». Puis plus rien. Trois petits tours et puis s'en vont. On se prend à penser qu'ailleurs, un tel événement aurait fait l'objet d'une distribution sur disque dans le mois suivant sa création. Nul n'est prophète..

Bien sûr la diffusion de l'événement par la Chaîne culturelle de Radio-Canada aura grandement contribué à étendre l'auditoire de l'œuvre, mais il reste mainte- 
nant un enregistrement, quelque part, qui attend d'être rendu disponible comme le mérite celui d'un tel événement, c'està-dire largement. C'est à cette seule condition que l'on pourra vraiment mesurer l'impact de cette soirée du 3 juin 2000.

Cet événement réunissant une très grande partie des forces vives de la musique d'ici aura-t-il gagné le pari de secover l'indifférence générale autour des musiques de création? Cela reste à vérifier. Une chose est sûre, c'est que cette soirée du 3 juin 2000 restera gravée dans la mémoire de ceux et celles qui eurent la chance de pouvoir y assister. Peut-être, pour les compositeurs, comme Michel Gonneville, constituera-t-elle, la réalisation ultime de l'Utopie:

[...] ce genre de moment qui, lorsque nous en serons aux compromis et poussés vers les magouilles, restera comme le souvenir d'un indispensable idéalisme moral, qui nous donnera mauvaise conscience si nous tentons de le repousser dans le fond de notre inconscient.

On ne peut, en définitive, que souhaiter que ce qui caractérisera la Symphonie du millénaire dans un avenir proche, ce sera d'avoir été le premier d'une série d'événements célébrant l'originalité de la création contemporaine dans notre coin de planète.

«ll a fait beau et le show était gratuit. Les compositeurs ont créé et ont enfin crié leur existence sur la place publique. On était là. Hourra!»

\section{Remerciements}

Je tiens à remercier chaleureusement Serge Arcuri, Walter Boudreau, Denys Bouliane, Vincent Collard, Yves Daoust, Alain Dauphinais, André Duchesne, Lovis Dufort, Sean Ferguson, Michel Gonneville, André Hamel, Alain Lalonde, Estelle Lemire, Jean Lesage, Luc Marcel, Marie Pelletier, John Rea, Anthony Rozankovic et Gilles Tremblay, pour le temps qu'ils ont consacré à répondre à mes questions. Je remercie également Michel Duchesneau de m'avoir permis cette exploration.

DUROZOI, G. et LECHERBONNIER, B. (1972), Le Surréalisme : théories, thèmes, techniques, Paris, Larousse, collection «thèmes et textes", 286 p. 